\title{
Polysèmes
}

Revue d'études intertextuelles et intermédiales

\section{Transpositions cinématographiques dans l'œuvre de Salman Rushdie}

\section{Elsa Sacksick}

\section{(2) OpenEdition}

\section{Journals}

Édition électronique

URL : http://journals.openedition.org/polysemes/680

DOI : 10.4000/polysemes. 680

ISSN : 2496-4212

Éditeur

SAIT

Édition imprimée

Date de publication : 1 janvier 2012

Pagination : 203-225

ISSN : 0999-4203

\section{Référence électronique}

Elsa Sacksick, «Transpositions cinématographiques dans l'œuvre de Salman Rushdie », Polysèmes [En ligne], 12 | 2012, mis en ligne le 01 mars 2015, consulté le 30 avril 2019. URL : http:// journals.openedition.org/polysemes/680; DOI : 10.4000/polysemes.680

Ce document a été généré automatiquement le 30 avril 2019.

Polysèmes 


\title{
Transpositions cinématographiques dans l'œuvre de Salman Rushdie
}

\author{
Elsa Sacksick
}

1 Dans l'œuvre de Salman Rushdie, écrivain migrant par excellence, la notion de transposition, qui consiste dans un premier temps à «faire passer ailleurs, ou dans un autre domaine ", ne peut qu'être cruciale. Si le terme de transposition n'apparaît pas explicitement sous sa plume, on trouve néanmoins celui, récurrent, de "translation ». Pris dans son acception anglaise, celui-ci présente l'intérêt de mettre en lumière les deux facettes inhérentes à la transposition. Outre son acception spatiale, la transposition induit en effet également l'idée de transformation puisqu'elle consiste à « faire changer de forme en passant dans un autre domaine ». Or "translation » renvoie d'une part au déplacement et au glissement dans l'espace mais aussi, à travers la notion de «traduction ", à l'idée de changement de forme. Rushdie établit d'ailleurs une relation directe entre ces deux notions puisqu'il insiste sur l'idée que la translation spatiale induit toute une série de transformations ou de traductions: traduction de l'être (ou de l'identité), de la lettre (ou de la langue) et traduction de la forme romanesque.

\section{Transpositions identitaires et linguistiques}

2 L'identité est en effet au cœur des processus de transposition. À l'image de leur auteur, les personnages dans les romans de Rushdie sont sans cesse soumis à des translations géographiques, ils voyagent, émigrent, se déplacent selon des axes verticaux et horizontaux ${ }^{1}$. Or les personnages translatés sont invariablement traduits, c'est-à-dire transformés, comme le constate le narrateur migrant de Shame : « I am a translated man, I have been borne across » (Shame $\left.{ }^{2} 29\right)$. Rushdie va même plus loin en faisant du migrant, figure emblématique de ses romans, un mutant.

3 C'est peut-être dans The Satanic Verses ${ }^{3}$ que Rushdie a exploré de la manière la plus approfondie l'art de la transposition. Les migrants y deviennent des êtres monstrueux, révélant à même leur chair, portant à même le corps, les effets de la transposition, comme si, en perdant la fixité dans l'espace, ils avaient aussi perdu la fixité de la forme. 
Outre l'exemple marquant des immigrés mutants parqués dans un hôpital ${ }^{4}$, Rushdie se focalise sur la transposition d'un personnage indien en citoyen britannique. Au cours de sa migration de l'Inde vers l'Angleterre, Saladin est transformé en animal, et plus précisément en bouc. Ces transpositions métamorphiques, pour lesquelles Rushdie utilise le terme de "transmogrifications $»^{5}$ - terme qui scelle étymologiquement le lien entre transformation et migration - parcourent tous ses romans. Qui plus est, The Satanic Verses est également fondé sur une transposition religieuse. Gibreel est «translated into an avatar of the archangel» (216), Saladin est transposé en personnage diabolique et tout le roman repose sur le déplacement d'un épisode coranique (les versets sataniques) dans une œuvre de fiction.

Le phénomène de «translation » n'affecte pas seulement l'identité mais aussi la langue, et le jeu des transpositions linguistiques pourrait à lui seul constituer un chapitre. Il faudrait parler de l'insertion de l'hindi dans l'anglais qui occasionne des traductions ou des impossibilités de traduction, tel le mot « Takallouf » qui se dérobe à la «translation » (spatiale et linguistique): "Takallouf is a member of that opaque, world-wide sect of concepts which refuse to travel across linguistic frontiers » (S 104).

5 Mais il faudrait aussi parler de la translation ou migration des catégories grammaticales, avec le processus de recatégorisation si cher à Rushdie. Les adjectifs sont, par exemple, transposés en verbes: " the train yellowed and browned its way " (Midnight's Children ${ }^{6}$ 443), ou en adverbes: "greenly-blackly she sails into my cell » (437), " her secrets are leaking saltily out of her eyes $»^{7}$ (MC 105). Il peut être intéressant de rappeler à ce propos que, en traductologie, ces recatégorisations grammaticales sont précisément appelées des transpositions.

6 Il est également impossible de passer sous silence la transposition somatique de la langue, c'est-à-dire sa traduction physique sur le corps qui s'impose comme une caractéristique de l'écriture rushdienne. Ainsi, dans les romans de Rushdie le langage s'incarne. Dans The Satanic Verses, le personnage de Saladin qui est cocu voit littéralement des cornes lui pousser sur le front ; ce même personnage au visage angélique devient l'ange déchu (c'està-dire le diable) au moment même où il tombe littéralement du ciel. De même, à la fin de Shame, le personnage de Sufiya, véritablement consumée par la rage, devient une boule de feu. Il y a donc chez Rushdie un échange entre figuré et littéral au cours duquel la lettre se fait chair, les expressions figurées sont transposées de manière littérale. La métaphore devient active, elle engage des métamorphoses.

7 Ainsi, tout au long de son œuvre, Rushdie s'interroge sur les effets de la migration, et explore les différentes formes de transposition qu'elle induit. Il est encore une autre forme de translation qui fera l'objet de notre analyse : celle qui va du texte à l'image. C'est ce passage entre littérature et art visuel, cette traduction de l'image dans l'écrit, que nous nous proposons d'explorer ici. Après avoir mis en évidence la part du cinéma dans les romans de Rushdie, nous verrons comment les techniques cinématographiques, et plus précisément celles du cadrage et du montage, sont inscrites dans l'écriture même, tant au niveau de la narration que de la syntaxe.

\section{La transposition cinématographique}

8 Le choix de la thématique cinématographique n'est pas fortuit mais suggéré par l'auteur lui-même qui ne cesse de réaffirmer sa dette par rapport au septième art. Il explique dans 
ses essais que le cinéma a considérablement influencé ses débuts, et cite Welles, Buñuel et Godard parmi ses sources d'inspiration ${ }^{8}$. C'est encore à la métaphore du cinéma qu'il a recours pour rendre compte de l'aspect insaisissable du réel : «Reality is a question of perspective. Suppose yourself in a large cinema, sitting at first in the back row and gradually moving up, row by row, until your nose is pressed against the screen. Gradually the stars' faces dissolve into dancing grain, the illusion dissolves, or rather it becomes clear that the illusion itself is reality " (MC 167). L'omniprésence du cinéma est indiscutable dans ses romans qui regorgent de films (dont une adaptation cinématographique de l'histoire de Mahomet dans The Satanic Verses), de personnages acteurs ou réalisateurs ${ }^{9}$, et elle semble d'ailleurs se renforcer de Midnight's Children à The Satanic Verses ${ }^{10}$.

9 L'auteur a de surcroît recours au champ lexical et aux techniques cinématographiques pour aider le lecteur à visualiser une scène. Il écrit par exemple : «I permit myself to insert a Bombay talking style close up [...]. I superimpose turbulent long-shots of street riots » (MC 346).

De même, dans The Satanic Verses, le narrateur s'aide de la technique du montage pour décrire la schizophrénie de Gibreel : « nifty cutting is required to pull off this double role, the two of them can never be seen in the same shot [...] trust technology with scissors and Scotch tape " $(108)^{11}$. Saleem a fréquemment recours aux techniques des bandes-annonces pour accrocher l'attention de Padma: «I resort to movie trailers. There is to be electrocution, a rain forest, a pyramid of heads [...]. [N]arrow escapes are coming, and a minaret that screamed! In short there are still next-attractions and coming-soons galore » (MC 346).

Rushdie propose même une transposition indienne du "Hollywood movie » et affiche clairement ses références aux codes de «Bollywood» : « this very melodramatic device of the babies being exchanged at birth which I liked because it was a genuine kind of Bombay-talkie, B-movie notion, and I thought that a book which grew out of a movie city ought to contain such notions $»^{12}$. L'utilisation des chansons dans The Satanic Verses, composante incontournable des «Bollywoods $»^{13}$, participe également de cette transposition du cinéma dans le roman. Rappelons à ce propos que l'attrait de Rushdie pour le cinéma est si fort qu'il ira jusqu'à franchir la frontière entre les deux arts. Après avoir transposé des effets cinématographiques dans l'écriture de Midnight's Children, il finira par adapter le roman pour la $\mathrm{BBC}^{14}$. Cette remarque permet d'esquisser une réflexion sur la différence entre l'adaptation et la transposition qui ne peut cependant pas être développée dans le cadre de ce travail. Contentons-nous d'avancer que l'adaptation implique un changement effectif de medium : il s'agit de la même œuvre mais en images, sur écran, et non plus en mots, tandis que la transposition consiste en la transformation interne de l'écriture qui est modifiée par la prégnance de l'image.

Cependant, Rushdie ne se contente pas d'emprunter au cinéma son vocabulaire ${ }^{15}$, il se sert aussi de ses techniques et de ses effets, comme celui du cadrage, et transpose la technique du montage au cœur de son écriture par le jeu particulier de la ponctuation.

\section{De l'image dans l'écrit : le cadrage}

13 La dimension visuelle de l'écriture rushdienne est indiscutable et tient, pour beaucoup, à la manière dont il décrit les scènes et à l'attention toute particulière qu'il porte à la 
notion de cadrage. Certaines scènes semblent donner toutes les indications nécessaires à un metteur en scène éventuel, car Rushdie s'ingénie à créer des plans larges ou serrés, fixes ou animés et à jouer sur la notion de hors champ.

La thématique du cadrage se trouve d'autant mieux adaptée à Midnight's Children que ce roman est structuré par le trope du trou, véritable cadre au travers duquel les personnages voient le monde. Celui-ci apparaît d'abord avec l'histoire fondatrice du rond découpé dans le drap qui délimite le cadre par lequel le grand-père médecin de Saleem ausculte et perçoit sa future femme. Il est ensuite décliné sous plusieurs formes tout au long du roman, avec, entre autres, le trou dans le voile ("châdor») de Jamila à travers lequel elle regarde le monde, ou encore les trous dans le panier en osier à travers lesquels Saleem assiste au strip-tease de sa mère. Ce trou fonctionne d'une certaine manière comme une métaphore de l'objectif de la caméra qui restreint le point de vue et n'offre que certains plans sur la réalité. Saleem joue d'ailleurs de manière récurrente sur cette notion et s'impose comme le personnage qui refuse le hors champ, lui qui ne souffre pas de laisser des récits hors champ et qui voudrait tout inclure dans sa narration.

La référence au cadrage devient explicite dans la scène où il épie sa mère et son amant Nadir. Il y renvoie d'ailleurs clairement, et avec humour, au septième art: "what I'm watching here on my dirty glass cinema-screen is, after all, an Indian movie " (MC 217) ; "so it was that life imitated bad art " (MC 217). La scène commence avec Saleem qui, comme souvent en position de voyeur, semble prendre place derrière la caméra: "pressing my nose against a corner of the grubby window-pane» (MC 216). Le plan est limité par l'encadrement de la fenêtre : «through the dirty, square, glassy cinema-screen of the Pioneer café's window, I watched Amina and [...] Nadir play their love scene » (MC 216-17). Puis vient un travelling suivi par un gros plan:

I followed her with my eyes as she went [...] past rickety tables [...]. I saw my mother sit down [...] and then I saw the man who rose to greet her.

The skin on his face hung in folds [...] his teeth were stained.

(MC 216)

16 Ce gros plan sur le visage est suivi d'une série de cadrages serrés. Le premier se focalise sur un paquet de cigarettes sur la table, et les suivants s'arrêtent sur les mains d'Amina et Nadir, qui représentent par métonymie leurs propriétaires :

But now hands enter the frame - first the hands of Nadir [...] hands flickering [...] creeping forward then jerking back; next a woman's hands [...] inching forward like elegant spiders [...] lifting up, off [...] hands hovering above three fives, beginning the strangest of dances. (MC 217)

L'évocation de ce ballet de mains résonne alors comme un hommage au film d'Ettore Scola « Le Bal », entièrement focalisé sur les pieds des danseurs.

Dans Midnight's Children, Saleem endosse clairement le rôle de cinéaste, il devient l'œil de la caméra, il maîtrise le cadrage et le drap troué, tandis que son grand-père le subissait : « condemned by a perforated sheet to a life of fragments [...]. I have done better than my grandfather, because when Aadam Aziz remained the sheet victim, I have become its master " (MC 121). Dans ce sens, parce que Saleem garde à la naissance les yeux ouverts pendant des mois : « the little chap never blinks! » (MC 125), il semble proposer une vision sur le mode du plan séquence, c'est-à-dire sans coupure ni montage : «a batless gaze » ( $M C$ 125). Il se rangerait alors derrière la théorie d'André Bazin sur le montage cinématographique, selon laquelle le cinéma est « une fenêtre ouverte sur le monde » et doit montrer la réalité sans coupure ni trucage, c'est-à-dire en limitant autant que possible les opérations de montage ${ }^{16}$. À travers ce refus apparent du montage et de la 
rupture apparait déjà un point fondamental dans le roman : le souci de continuité qui oppose Midnight's Children à The Satanic Verses.

\section{Traduction des techniques cinématographiques dans l'écriture romanesque : le montage narratif et syntaxique}

La prégnance du cinéma dans l'œuvre de Rushdie va plus loin que la qualité visuelle de l'écriture et l'utilisation des notions de cadrage dans les descriptions. Certaines techniques cinématographiques, notamment le montage, sont en effet inscrites dans l'écriture même de l'auteur, au point d'en refaçonner la syntaxe et la narration. Nous reverrons dans un premier temps la structure narrative globale sous l'angle du montage, c'est-à-dire comment les récits sont montés les uns par rapport aux autres, puis nous nous tournerons vers les choix syntaxiques et plus précisément vers la ponctuation, qui permet de se pencher, à plus petite échelle, sur l'enchaînement des plans. Il apparaîtra alors que les choix de ponctuation déterminent un type de montage bien particulier et permettent par exemple de transposer dans l'écriture les techniques du raccord et du faux raccord. La notion de montage permettra en outre de mettre en évidence une évolution du style rushdien entre Midnight's Children et The Satanic Verses. En étudiant la construction narrative par le biais du septième art, dont Rushdie se réclame, il apparaît que deux types de montage sont mis en jeu dans les deux romans. Tandis que Midnight's Children relèverait plutôt d'un montage parallèle et continu, The Satanic Verses correspondrait à un montage convergent et discontinu.

\section{Midnight's Children et le montage parallèle continu}

Ainsi que nous l'avons indiqué précédemment à propos du montage minimal que Saleem feint de privilégier, Midnight's Children est un roman sous le signe de la fluidité, tant narrative que syntaxique, si bien que, pour reprendre le vocabulaire cinématographique, il relève d'un "montage continu ». Sa structure narrative globale est d'autant plus fluide qu'elle est linéaire puisque fondée sur un déroulement chronologique (la vie de Saleem de sa conception à sa mort). En revanche, si le passage entre les récits, c'est-à-dire l'agencement narratif global, est assez linéaire, une microlecture révèle que la transition entre les scènes, qui équivaudrait au montage des plans, joue sur l'alternance. L'auteur excelle en effet à mettre des scènes en parallèle et à les développer en alternance sans les faire forcément converger l'une vers l'autre, si bien qu'il semble faire dans ce roman le choix d'un montage parallèle continu.

Le passage entre deux scènes montées en parallèle s'effectue selon deux techniques syntaxiques propres à Midnight's Children. Il s'agit d'une part de l'utilisation récurrente de la structure syntaxique parallèle "while $»^{17}$ qui permet de signaler la transition entre deux actions concomitantes, et d'autre part de l'utilisation surprenante et proliférante des points de suspension qui figurent sur presque toutes les pages du roman. Ces derniers servent souvent à effectuer un changement de scène et donc, cinématographiquement parlant, de plan. Ce ponctème est propre à Midnight's Children, comme les deux points sont, nous le verrons, la marque propre de The Satanic Verses. Il revêt une fonction assez visuelle dans la mesure où il permet par exemple de signaler une transition au ralenti 
entre deux plans. Dans l'exemple suivant, les points de suspension s'apparentent à un lent mouvement de caméra qui balaye la scène sur le modèle du plan panoramique :

He turns from the window...

... To see Naseem weeping into a pillow. (MC 33, les

points de suspension sont dans le texte)

Le retour à la ligne complète ainsi visuellement le mouvement de rotation du personnage. Les points de suspension introduisent dans le roman une transition fluide et permettent, selon les termes de Jacques Dürrenmatt, «la production de phrases élastiques, aux articulations et aux frontières indécises $»^{18}$, tout à fait en accord avec la qualité protéiforme et fluide du roman de Rushdie.

Tout au long de Midnight's Children le narrateur jongle entre de multiples récits. Deux, trois, voire quatre récits sont mis en parallèle sur la page et montés en alternance grâce à l'utilisation combinée de ces deux procédés en début de phrase. Le principe du montage parallèle alterné de deux scènes culmine au chapitre six, « Many headed monsters », dans lequel le narrateur présente en parallèle le trajet de sa mère qui se rend chez le prophète et celui de son père qui va payer sa rançon au vieux fort ${ }^{19}$. Tout le chapitre se construit sur l'évolution simultanée des deux personnages et sur un glissement visuel d'une scène à l'autre, matérialisé par les deux mêmes procédés :

she follows him into a darkened doorway while her face bursts into flames.

... While at the Old Fort, Ahmed Sinai waits (MC 82, les points de suspension sont dans le texte)

I must admit it: to her shame, my mother screamed...

... While at the Old Fort, monkeys scream among ramparts.

(MC 84, les points de suspension sont dans le texte)

Les points de suspension pourraient alors évoquer le flou de transition utilisé dans certains films à l'eau de rose (notamment les séries B produites à Bollywood) tandis que la conjonction «while» peut rappeler le principe des «soap» qui mettent systématiquement en parallèle plusieurs intrigues. Ce type de transition syntaxique et narrative, s'il devait être porté à l'écran, pourrait également être transcrit par un fonduenchaîné. Il apparaît donc que ces deux procédés contribuent à renforcer la continuité narrative au lieu de jouer sur la rupture habituellement associée à l'alternance.

\section{The Satanic Verses et le montage convergent discontinu}

Dans The Satanic Verses, Rushdie a fait un choix tout différent dans la mesure où le principe d'alternance génère une esthétique de la discontinuité et où le montage parallèle laisse place à un montage convergent. Directement opposé à la linéarité de Midnight's Children, The Satanic Verses se construit sur des ruptures temporelles (vingtième siècle, septième siècle, temps de l'Hégire), des ruptures spatiales (les personnages voyagent de Bombay à Londres en passant par Jahilia, qui se pose comme la transposition de Médine) et sur des ruptures narratives. Le roman est nettement divisé entre les chapitres au vingtième siècle traitant des aventures de Gibreel et Saladin et les chapitres oniriques " coraniques $»^{20}$. L'alternance est d'autant plus claire qu'elle suit l'ordre pair / impair ${ }^{21}$. Les chapitres impairs sont eux-mêmes subdivisés entre les parties traitant des aventures de Saladin et de celles de Gibreel, si bien que la structure narrative de The Satanic Verses 
obéit globalement à la figure du tressage et joue sur l'interruption et l'entrecoupement. En outre, tandis que Saleem s'engage souvent dans deux ou trois récits en parallèle qu'il fait néanmoins se rejoindre en de rares occasions, les deux récits principaux de The Satanic Verses alternent régulièrement et se développent en parallèle jusqu'à se rejoindre, selon la technique du montage convergent que Deleuze définit ainsi : «faire alterner les moments de deux actions qui vont se rejoindre. Et plus les actions convergent, plus la jonction approche, plus l'alternance est rapide $»^{22}$.

Ainsi, dans The Satanic Verses, deux récits concurrents (l'un traitant de Gibreel, l'autre de Saladin, deux personnages en compétition) deviennent concourants ou convergents. Sans cesse différée, leur rencontre est pourtant annoncée comme inexorable tout au long du roman $^{23}$. The Satanic Verses s'oppose alors nettement à Midnight's Children dont la structure ou le montage narratif n'est pas gouverné(e) par l'alternance entre deux récits concourants pour la simple raison que Saleem n'admet pas de concurrent. La narration de Midnight's Children n'est donc pas diffractée en deux grands récits ${ }^{24}$. Les innombrables récits qui traversent ce roman sont absorbés et enchâssés à l'intérieur du récit de Saleem qui se pose comme la voix unique et unificatrice du roman.

Par ailleurs, ce montage convergent qui marque la structure narrative générale de The Satanic Verses est également pertinent à plus petite échelle, au niveau des plans ou des séquences. Il apparaît en effet que la scène de convergence des deux récits, c'est-à-dire la rencontre tant attendue entre Gibreel et Saladin, est traitée sur un mode nettement visuel. Le narrateur fait alterner les plans sur Saladin avec ceux sur Gibreel pour les faire converger, sur le mode du champ / contre-champ, jusqu'à leur rencontre effective ( $\mathrm{SV}$ 424 puis 428$)^{25}$.

Si cette scène s'inscrit dans un contexte visuel, et il faudrait dire ici télévisuel, c'est en outre parce que le narrateur procède à une véritable mise en scène, la description évoquant par certains aspects la retransmission télévisée d'un événement sportif ${ }^{26}$. Il adopte en effet le ton parodique et enthousiaste du commentateur sportif (les interjections et le présent de description y travaillent activement) en décrivant le terrain et les joueurs déjà en place : "Shepperton! - Pamela and Jumpy are already there [...] when Chamcha [...] arrives » (SV 421) et «Why, here's Stucconia of the Veneerings, those brand-new, spick and span people » (SV 422). Puis il décrit l'entrée en scène de Saladin : "Chamcha enters the arena ; and is amazed ( $S V$ 422). Suit alors une série de verbes au présent qui retracent la progression du protagoniste sur le terrain : «Chamcha [...] spots Gibreel. Yes! there on London Bridge which is of Stone, without doubt! Gibreel! And that must be Alleluia, his Icequeen Cone! Battutta to his left, Sisodia at Allie's right " (SV 422-23).

Le commentaire journalistique se poursuit avec des phrases saturées de ruptures syntaxiques et typographiques (points d'exclamations, alinéas, tirets, points virgule) qui peuvent être interprétées visuellement par des changements de plans abrupts: "Chamcha struggles through the crowd", "but he is resolved - Gibreel! he will reach Gibreel! ... - Chamcha, off balance, staggers back, is crushed [...] and, to save himself retreats within " (SV 422-23). Dans ces exemples, comme dans les pages qui suivent, le lecteur peut imaginer la caméra se déplacer entre les différents personnages et les multiples actions. Cet extrait rend donc compte du mouvement que Rushdie s'efforce de donner à la description et constitue un exemple d'hypotypose. L'énergie du discours rushdien transmue ses descriptions en images cinétiques, presque cinématographiques l'écriture déborde sur le cinéma. 

se répercute également sur la structure syntaxique. Les phrases courtes et les syncopes syntaxiques évoquent une suite de plans courts qui s'enchaînent à un rythme rapide et saccadé, et renvoient de ce fait à un montage filmique très découpé. Ici encore, c'est l'utilisation particulière de la ponctuation dans le roman qui contribue largement à créer cet effet et qui pourrait fonctionner comme des indications techniques de l'auteur quant au montage des plans. L'utilisation des tirets, des parenthèses, des points-virgules, c'està-dire de tout ce qui peut venir interrompre le déroulement syntagmatique, est frappante dans le roman et particulièrement dans cette scène de convergence des deux personnages ${ }^{27}$. Il faut encore ajouter à cela la sur-représentation des deux points qui opèrent un montage textuel particulier.

Ce ponctème, caractéristique de The Satanic Verses, opère un passage abrupt qui renvoie à la technique du collage (d'un point de vue syntaxique autant que visuel). Les deux points mettent en place une juxtaposition directe, une mise en relation brutale de deux énoncés. Ils instaurent un passage fulgurant, par bonds, en opposition directe avec les points de suspension qui évoquaient une typographie du glissement et un montage fluide. Des phrases telles que : «Gibreel: the dreamer, whose point of view is sometimes that of the camera " (SV 108), «Saladin: was not dead but weeping ( SV 131), ou : «He's coming: making his way up Cone Mountain » (SV 92) mettent directement les personnages devant les yeux avec la neutralité d'une didascalie. L'omniprésence des deux points dans les chapitres coraniques est frappante, peut-être parce que, avec leur effet de collage brut, ils font surgir un pan de la phrase et créent un effet d'annonce, voire, dans ce contexte religieux, d'annonciation: «Walk quickly, out of this wrong place, this underworld. God: no escape » (SV 418), ou "The Imam sends a message of his own: and summons, conjures up Gibreel » (SV 211). La phrase qui présente le personnage de Mahound: «The businessman: here he comes " (SV 92) crée de surcroît un effet «bande-annonce », et l'utilisation du présent, propre aux chapitres coraniques et oniriques, amplifie encore l'effet en créant des sortes de vignettes qui évoquent l'écriture du scénario ou même du story-board.

31 Ainsi, l'utilisation excessive des deux points ouvre une brèche dans la continuité de la phrase et induit une juxtaposition abrupte de deux propositions, qui pourrait être rendue visuellement par une saute ou un insert, c'est-à-dire une juxtaposition directe de deux plans. Les deux points indiqueraient alors visuellement un hiatus dans la continuité des plans et peuvent jouer à faire surgir un gros plan dans un plan d'ensemble, ou à insérer brutalement un plan fixe dans un travelling. La description de Mahound, par exemple, le fait surgir dans un plan d'ensemble. Le personnage est vu de loin au milieu de la foule puis le point de vue saute à un plan américain (le même homme vu de plus près): " among them, one wanders [...] apart from the cheerful crowd: a tall man in loose white robes [...] his beard is shaped " (SV 95). Si la transition entre les phrases devait être mise en images dans The Satanic Verses, le montage entre les plans procéderait par collages ou coupes plutôt que par fondu enchaîné. L'esthétique cinématographique de ce roman est donc bien loin des transitions fluides ménagées par les points de suspension dans Midnight's Children.

Pour finir, c'est peut-être lors de la scène de la mort de Rosa que l'exemple le plus pertinent d'un montage cinématographique discontinu est mis en place. Différentes versions d'un même événement (la rencontre entre Rosa et Martin de la Cruz puis le meurtre de Martin) entrent en concurrence avec un montage alterné et heurté qui 
évoque la technique développée dans le film d'Alain Resnais Smoking, No Smoking. À partir des mêmes images, le narrateur propose différents montages narratifs qui donnent lieu à des interprétations opposées : Rosa est séduite par Martin, ou le repousse avec pruderie ; ce dernier est tué par Aurora, ou par Rosa, ou par son mari. La scène s'ouvre sur des références visuelles et le montage évoque d'abord le procédé du fondu enchainé: " something happened, the scene grew blurred, one minute they were lying [...] - while the next minute she was recoiling from him [...] the two possibilities kept alternating " ( $S V$ 152). Or ces différentes versions sont montées les unes après les autres. Le montage des scènes devient alors de plus en plus incohérent et le rythme de plus en plus heurté, les versions contradictoires se succèdent par sautes et inserts du fait de la présence des tirets :

now Don Enrique drew his small pistol and aimed at his rival's heart

-and he felt Aurora stabbing him in the heart [...]

-and he felt his victim's knife entering his heart, as Rosa

stabbed him [...]

-and after Henry's bullet had killed him the Englishman took

the dead man's knife and stabbed him. (SV 155, c'est moi qui souligne)

Néanmoins, contrairement à Alain Resnais qui construit sa narration sur un montage contrastif (le mode du « ou bien... ou bien » régit la succession des scènes), Rushdie, dans une logique de surenchère, choisit ici un montage intensif, voire exhaustif, car les différentes alternatives s'ajoutent les unes aux autres sans s'exclure (sur le mode du « et... et ...et »).

L'écriture de Rushdie dans The Satanic Verses est visuelle et cinétique, l'image tressaute (trouvant un équivalent sonore dans le bégaiement des personnages), elle met devant les yeux du lecteur des plans sans continuité qui s'enchaînent de manière abrupte, sur le mode du zapping, à propos duquel Saladin propose une réflexion (SV 405). En d'autres termes, au moyen des deux points, des points virgules ou des tirets, par exemple, Rushdie affiche la rupture. La transition (lors des changements de plan) devient visible et s'exhibe au lieu de s'effacer au nom de la cohérence et de la continuité. Dans The Satanic Verses, ce n'est plus la loi du raccord, de la transition fluide et du montage minimal qui prime. Rushdie semble opter alors pour une esthétique contemporaine avec une technique de montage discontinu et heurté qui rappelle la «nouvelle vague » et plus précisément la saute godardienne et le faux raccord. Les deux techniques visaient à briser la continuité et la fluidité des plans, donc l'unité filmique, tout en faisant participer activement le spectateur. de transposition. Rushdie transpose la technique du raccord grâce aux points de suspension et celle du faux raccord grâce aux deux points. La ponctuation devient emblématique de l'écriture, elle opère un montage syntaxique et correspond à un type de montage visuel particulier: Midnight's Children propose une écriture des points de suspension, du glissement et du fondu enchaîné et The Satanic Verses une écriture des deux points, de la saute et du collage.

Deux citations liées au montage cinématographique pourraient synthétiser cette différence d'esthétique. Si le narrateur de The Satanic Verses revendique une esthétique du montage et de la discontinuité : "Trust technology with scissors and Scotch tape » (SV 108), celui de Midnight's Children préfère les codes du mélodrame et une fluidité formelle :

I leave you with the image of a ten-year-old boy; zooming out slowly into a long-shot, I allow the sound-track music to drown my words [...] It rolls along, rolls along, rolls along ... (Fade 
out).

(MC 237)

L'analyse du montage dans les deux romans nous amène de ce fait à mieux cerner la différence stylistique entre Midnight's Children et The Satanic Verses. De son premier à son troisième roman, Rushdie tend vers une accentuation de la discontinuité, The Satanic Verses présentant une structure plus éclatée, à l'image de l'identité schizophrène des personnages. Qu'il s'agisse de la syntaxe ou de la narration, le roman prend une forme plus heurtée. Cette tendance s'accentue encore dans les romans suivant The Satanic Verses et s'accompagne d'une certaine violence. Les translations dans l'espace ressemblent moins à des glissements qu'à des chutes, la traduction identitaire du migrant présente un caractère plus radical et pessimiste, la transposition du migrant est plus douloureuse (schizophrénie de Gibreel, métamorphose bestiale et monstrueuse de Saladin, folie dans Shame, et meurtre dans The Moor's Last Sigh). Au contraire, dans Midnight's Children, Saleem refusait en quelque sorte la traduction et la translation vers une identité pakistanaise puisqu'il devenait amnésique lors de son passage au Pakistan.

Pour en revenir à la transposition cinématographique, Rushdie retravaille l'écriture à partir du cinéma, il propose dans ces deux romans une passerelle entre l'écrit et le visuel. Avec une écriture façonnée par et sur le mouvement, Rushdie ne pouvait que se tourner vers l'art cinétique par excellence : le cinéma. Le choix de ce medium n'est pas fortuit mais éminemment adapté à la thématique de la migration. Rushdie se charge de révéler le lien qui court entre le migrant et le septième art lorsqu'il explique à propos du migrant et de son rapport imaginaire à la réalité :

For the plural, hybrid, metropolitan result of such imaginings

[the migrant's necessity for new imaginative relationship with

reality], the cinema, in which peculiar fusions have always

been legitimate [...] may be the ideal location. ${ }^{28}$

\section{BIBLIOGRAPHIE}

Deleuze, Gilles. L'image-mouvement. Paris : Éditions de Minuit, 1983.

Dürrenmatt, Jacques. Bien coupé, mal cousu. De la ponctuation et de la division du texte romantique.

Paris : PU de Vincennes, 1998.

Rushdie, Salman. Imaginary Homelands. London: Granta Books, 1991.

Rushdie, Salman. Midnight's Children. London: Vintage, 1981.

Rushdie, Salman. Shame. London: Vintage, 1983.

Rushdie, Salman. Step across this Line. London: Jonathan Cape, 2002.

Rushdie, Salman. The Satanic Verses. London: Vintage, 1988.

Rushdie, Salman. "Midnight's Children and Shame by Salman Rushdie”. Kunapipi 7 (1985).

Tadié, Alexis. « Salman Rushdie et The Satanic Verses : le questionnement des catégories ». Ateliers 2 (1995), Numéro spécial Salman Rushdie : 53-65. 


\section{NOTES}

1. Voir les translations horizontales de la marge vers le centre dans Shame avec les personnages d'Omar et de Sufiya, ou du centre vers la périphérie avec le personnage de Saleem dans Midnight's Children ou encore les translations sur des axes verticaux avec le rôle central du monte-charge dans Shame ou les plongées en enfer et les folles envolées de Gibreel dans The Satanic Verses.

2. Salman Rushdie, Shame, London: Vintage, 1983. Ci-après désigné comme $\mathrm{S}$ dans les références entre parenthèses dans le texte.

3. Salman Rushdie, The Satanic Verses, London: Vintage, 1988. Ci-après SV dans les références entre parenthèses.

4. Voir la description des mutants dans The Satanic Verses, op. cit., 167 à 171.

5. Voir The Satanic Verses, ibid., 34 et 282 et Midnight's Children, London: Vintage, 1981, 257.

6. Salman Rushdie, Midnight's Children. London: Vintage, 1981. Ci-après MC.

7. Rushdie affectionne également le passage vers le participe passé : «a stethoscoped return », ( Midnight's Children 11), « his travelled eyes » (MC 11), « Nonplussed A » (MC 22).

8. Voir Imaginary Homelands, London: Granta Books, 1991, 276.

9. On peut citer Hanif dans Midnight's Children et Sisodia et Hal Valence dans The Satanic Verses. Gibreel est d'ailleurs décrit de la sorte : « a creature of surfaces, like a movie screen » (SV 27).

10. Tandis que Midnight's Children commence avec l'ancêtre du cinéma, avec la boîte de Lifafa Das où l'on fait tourner manuellement les photos image par image (voir 75), The Satanic Verses laisse place aux superproductions avec force effets spéciaux.

11. Voir aussi dans ce roman, 216 ou 454-457, lorsque le narrateur fournit des indications de montage et donne l'illusion d'une prise de vue et de son en direct : « - Cut - a man lit by a sungun speaks rapidly into a microphone [...] - Cut - here is a brightly lit video store - Cut - Here is a serious head bathed in sunlight: a studio discussion » (SV 454-56).

12. "Midnight's Children and Shame by Salman Rushdie", Kunapipi 7 (1985). Kunapipi est une revue australienne spécialisée dans les nouvelles littératures et écrivains du Commonwealth.

13. Voir l'analyse d'Alexis Tadié dans «Salman Rushdie et the Satanic Verses : le questionnement des catégories ", Ateliers 2 (1995), Numéro spécial Salman Rushdie, 61. L'imbrication du cinéma dans le roman se complète lorsqu'on apprend que la chanson « My shoes are Japanese » est tirée d'un film de Raj Kapoor, voir Tadié, op. cit., 62.

14. Il écrit en effet le scénario pour la $B B C$ et participe à la réalisation du téléfilm, voir le dernier recueil d'essais de Rushdie : Step across this Line, London: Jonathan Cape, 2002.

15. Voir par exemple les termes techniques qui aident le lecteur à visualiser la scène dans l'exemple de Midnight's Children cité plus haut : «I permit myself to insert a Bombay talking style close up [...]. I superimpose turbulent long-shots of streets riots » (MC 346, soulignements ajoutés). Voir aussi The Satanic Verses 108, 216, 454.

16. Un pari esthétique que Saleem, pourtant obsédé par la volonté de tout inclure dans son récit, va se résoudre à abandonner : «nobody can face the world with his eyes open all the time » (MC 125).

17. Voir 18-19, 28 à 84, 148 et 342-43, ou même dans The Satanic Verses, 360.

18. Jacques Dürrenmatt, Bien coupé, mal cousu : de la ponctuation et de la division du texte romantique, Paris : PU de Vincennes, 1998, 46.

19. La séquence de montage alterné commence ainsi : « One journey began at a fort, one should have ended at a fort and did not ", " During one journey, monkeys danced entertainingly, while in the other place, a monkey was also dancing but with disastrous results » (80). 
20. Les chapitres réels et oniriques sont nettement distincts par la typographie mais aussi par l'utilisation des temps: le présent dans les chapitres oniriques et le passé dans les autres chapitres.

21. Les chapitres pairs traitent systématiquement de la réalité et les chapitres impairs des rêves. 22. Gilles Deleuze, L'image-mouvement, Paris : Éditions de Minuit, 1983, 49.

23. L'auteur multiplie les indices de convergence, et insiste sur l'imminence de leur rencontre tout au long du roman et dès leur séparation.

24. On aurait pu attendre une alternance entre les deux adversaires, Saleem et Shiva.

25. Voir par exemple l'alternance des plans 425 et la comparaison entre les deux personnages 426-27.

26. Le narrateur va même jusqu'à intégrer des chansons qui évoquent celles des supporters dans les gradins.

27. Voir, entre autres, cette phrase : «Gibreel, for all his stage-name and performances; and in spite of born-again slogans, new beginnings, metamorphoses; - has wished to remain, to a large degree, continuous » (SV 427).

28. Imaginary Homelands, op. cit., 125.

\section{INDEX}

oeuvrecitee Midnight's Children, Satanic Verses (The), Shame 